\title{
Behavioral Responses to Multisensory Stimulation in Preterm Infants
}

\author{
Kanagasabai PS ${ }^{1}$, Mohan $\mathrm{D}^{2}$, Lewis $\mathrm{LE}^{3}$, Rao BK ${ }^{4}$
}

${ }^{1}$ Mrs. Parimala S Kanagasabai, MPT, PhD candidate, School of Physiotherapy, University of Otago, New Zealand, ${ }^{2}$ Mrs. Divya Mohan, MPT, Assistant Professor, Department of Physiotherapy, School of Allied Health Sciences, Manipal University, India, ${ }^{3} \mathrm{Dr}$. Leslie E Lewis, MBBS, DCH, DNB, Professor, Department of Paediatrics, Kasturba Medical College, Manipal University, India, ${ }^{4} \mathrm{Dr}$. Bhamini K Rao, PhD, Professor, Department of Physiotherapy, School of Allied Health Sciences, Manipal University, India.

\section{Address for correspondence:}

Divya Mohan, Assistant Professor,

Department of Physiotherapy,

School of Allied Health Sciences, Manipal University,

Karnataka, India,

Tel: +919886794027

E-mail: dimspt2002@yahoo.co.in

Acknowledgements: We would like to thank the preterm infants and their parents for participating in our study. We also thank MrHariPrakash and Mr. Ayas Mohammed, Department of Speech and Hearing, Manipal College of Allied Health Sciences for helping us with the assessment of noise level in NICU and calibrating our auditory stimulation instrument for multisensory stimulation.

Funding: Nil

Conflict of Interest: The authors declare no conflict of interest. The work has been attributed to Department of Physiotherapy, School of Allied Health Sciences, Manipal University.

Permission from IRB: Yes

Ethical dilemmas faced during study: No

\section{How to cite}

Kanagasabai PS, Mohan D, Lewis LE, Rao BK. Behavioral Responses to Multisensory Stimulation in Preterm Infants. J Nepal Paediatr Soc 2016;36(2):110-114.

doi: http://dx.doi.org/10.3126/jnps.v36i2.14850

This work is licensed under a Creative Commons Attribution 3.0 License.

\section{(c) (i)}

\begin{abstract}
Background: Behavioral responses of preterm infant to sensory stimulation in the Neonatal Intensive Care Unit (NICU) are important to understand. This would enable NICU professionals to be cautious of overloading stimulations and ensure stability in preterm infants. The aim of this study was to describe the range of behavioral responses associated with multisensory (auditory, visual, tactile and vestibular) stimulation in preterm infants. Material and Methods: We recruited twenty-five preterm infants born at 28 to 36 weeks and birth weight of 1000 to 2000 grams for the study using convenience sampling. In the NICU, preterm infants from 32 weeks gestational age received multisensory stimulation session for duration of 12 minutes per day until discharge along with routine NICU care. During the stimulation, the behavioral responses of the preterm infants were observed and documented. Results: Limb activity and yawning were the most frequently observed behaviors. Sixty-five percent of the behavioral responses were observed during tactile stimulation. The frequency of observed behavioral responses decreased with successive stimulation sessions, which could indicate habituation responses in preterm infants to multisensory stimulation. Conclusions: Preterm infants showed behavioral responses indicating improved alertness and ability to integrate sensory stimulus. However, interpretation of preterm behaviors is a challenge because these behavioral responses could also indicate stress in preterm infants when coupled with other physiological and behavioral cues. Further studies are required to provide a detailed evidence for better clarity of infant's responses to environmental stimuli.
\end{abstract}

Key words: Behavioral response; Multisensory stimulation; NICU; Preterm infants.

\section{Introduction}

Un nlike the intrauterine environment, preterm infants nurtured in Neonatal Intensive Care Unit (NICU) are exposed to different sensory exposures. Preterm infants respond to this unnatural environment through various communicating behaviors ${ }^{1}$. Various theories are proposed in order to understand these behavioral 
responses. According to the Synactive Theory of Development these behaviors appear through interaction of five body subsystems that are interdependent. When the preterm infants are not able to adapt to the inappropriate environmental stimulation, they exhibit stress behaviors potentially indicating subsystem disorganization. These behaviors may be defense or avoidance behaviors, which are expressed through autonomic, motor, state, attention and interaction subsystems. Moreover, preterm infants are also reported to exhibit self-regulatory behaviors when they can successfully adapt to the environmental stimulation indicating stability ${ }^{2}$. Stress behaviors such as yawning, finger splay and hiccups have been observed in preterm infants during exposure to NICU environment (for example, sudden noise, bright light) and common NICU procedures such as endotracheal suctioning, chest physiotherapy, diaper change, nasogastric feeding and bathing and positioning ${ }^{3,4,5}$. In addition, preterm infants have also been reported to exhibit engagement and disengagement cues during interaction with the parents, caregivers or environment. For example, an infant with engagement cues is positively interacting with the parent and an infant with disengagement cue is moving away from the interaction. Both engagement and disengagement cues are considered coping behaviors and an opportunity for infants to learn how to regulate their social interaction behaviors. Further, based on these cues the parents could modify their behavior towards her infant ${ }^{6,7}$.

Overloading of sensory stimulations in NICU appears to negatively affect the fragile physical condition of preterm infants and immature organ systems, which could result in maladaptive motor behaviors $^{8,9}$. Whereas, environmental modifications and interventions facilitating positive stability and selfregulatory behaviors such as sucking, tucking, and hand to mouth maneuvers in preterm infants assist to reduce stress in preterm infants especially during handling for NICU procedures ${ }^{10}$. Therefore, positive sensory interventions such as massage therapy, multisensory stimulation, kinestheic stimulation are encouraged in NICU owing to the beneficial effects on behavioral state regulation,weight gain and development in preterm infants. However, there is limited evidence indicating physiological and behavioral readiness of preterm infants to such multisensory exposures ${ }^{11,12}$. Thus, it is important to understand the behaviors of preterm infants to such stimulations, which are dependent on physiological and autonomic stability of preterm infants. The aim of this study is to describe the frequency of behavioral responses of preterm infants to a multisensory stimulation that included auditory, visual, tactile and vestibular stimulations in the NICU.

\section{Material and Methods}

Twenty-five preterm infants admitted to NICU of Kasturba Hospital, Manipal were recruited for the study based on the selection criteria. The inclusion criteria were infants born between 28-36 weeks of gestation and birth weight ranging from 1000-2000 grams as such infants are more likely to spend longer duration in NICU to allow appropriate weight gain before discharge. The exclusion criteria were physiologically unstable preterm infants and infants with congenital anomalies or diagnosed central nervous system injury. The study protocol was approved by the Manipal University Ethics Committee and informed consent was sought from the parents (either mother or father) of preterm infants.

Demographic data of the preterm infants were collected from the medical record, which included gestational age, birth weight, length, head circumference, APGAR score at 1 minute and 5 minutes. The preterm infants were given multisensory stimulation when they reached a gestational age of 33 weeks because of better nervous system and maturity of involved sensory pathways after this age ${ }^{13}$. For infants born at 33-36 weeks, stimulation was started after 48 hours of birth. The stimulations were given during quiet alert state of preterm infant approximately 30 minutes before feeding. Stimulations were given for duration of 12 minutes daily, 5 days per week for a period of 10 days. The following multisensory stimuli were given:(a) Auditory stimulation, soft lullaby was played between (45-55 dB) for 3 minutes using a miniature speaker and an mp3 player positioned in the infants open crib approximately at a distance of 1 feet from the infant's ears; (b) Visual stimulation, a black and white visual stimulation card was hung at a distance of 8-10 inches from the infant for three minutes;(c) Tactile stimulation, gentle moderate pressure linear stroking massage was performed on infant for 3 minutes in the sequence of fore head, chest, abdomen, upper limbs and lower limbs in supine position; (d) Vestibular stimulation, gentle horizontal and linear rocking was given for 3 minutes. During tactile and vestibular stimulation face to face contact with the preterm infants was maintained.

The behavioral responses to the stimulations were observed and coded. The behaviors coded included limb activity (defined as extension and flexion of upper limbs and lower limbs), yawning, finger splay, tongue 
protrusion, gaze aversion, startles, tremors, hiccups and crying as these are common preterm behaviors. While administering the multisensory stimulations, physiological responses were observed for deviations in the vital signs using multi-parameter monitor (PHILIPS IntelliVue MP 20, India). Abnormal deviation in physiological parameters was considered as heart rate $(\mathrm{HR})>200$ or $<100$ beats per minute, respiratory rate $(R R)>20$ over baseline and oxygen saturation $\left(\mathrm{SpO}_{2}\right)$ below $86 \%$ for more than 15 seconds ${ }^{14}$. In order to prevent overloading of the sensory inputs, particular sensory stimulation was stopped with observation of negative behaviors (e.g. hiccups, tremors, startles and crying) or deviations in physiological parameters. Descriptive statistics were used to report the behavioral responses to multisensory stimulation.

\section{Results}

Twenty-five preterm infants were recruited for the study. The mean gestational age of preterm infants was 32.7 weeks. Mean birth weight was $1,445.4 \mathrm{~g}$ with a range of 1030-2000 grams. The Apgar score at 1 minute ranged from 4 to 8 and at 5 minute ranged from 8 to 10 . We documented 250 observations of behavioral responses to multisensory stimulation in the preterm infants over a period of 10 days. The multisensory stimulation was withheld in two infants for a period of 4 days because one of the infants was intubated during the study period due to hypoglycemia and respiratory distress and the other infant had frequent intense cry associated with hypoglycemia.

Table 1 illustrates the frequency of coded behaviors observed with multisensory stimulation. The most frequently observed behavior was limb activity, which accounted for $40.3 \%$ of response followed by yawning $(21.7 \%)$. Behaviors such as tremor, hiccups, gaze aversion, crying were the least frequently observed behaviors (occurring less than 5\%). Majority of behavioral stress signs were observed with the tactile stimulation $(65 \%)$ followed by vestibular stimulation $(28 \%)$ and least with auditory and visual stimulation.

Figure 1 illustrates the frequency of behavioral responses associated with the different types of stimulation used in the study. A gradual reduction in the frequency of behaviors was observed during the later stimulation sessions as compared to the initial sessions. The vital signs of preterm infants were within normal range, which indicates that they were physiologically stable during stimulation.
Table 1: Frequency and percentage of behavioral responses to multisensory stimulation in preterm infants $(n=25)$.

\begin{tabular}{lc}
\hline Behavioral response & Frequency, $\mathbf{n}(\%)$ \\
\hline Limb activity & $50(40.3)$ \\
\hline Yawning & $27(21.7)$ \\
\hline Tongue protrusion & $8(6.45)$ \\
\hline Finger splay & $12(9.67)$ \\
\hline Startle & $8(6.45)$ \\
\hline Tremor & $6(4.83)$ \\
\hline Hiccups & $6(4.83)$ \\
\hline Gaze aversion & $3(2.41)$ \\
\hline Crying & $4(3.22)$ \\
\hline
\end{tabular}

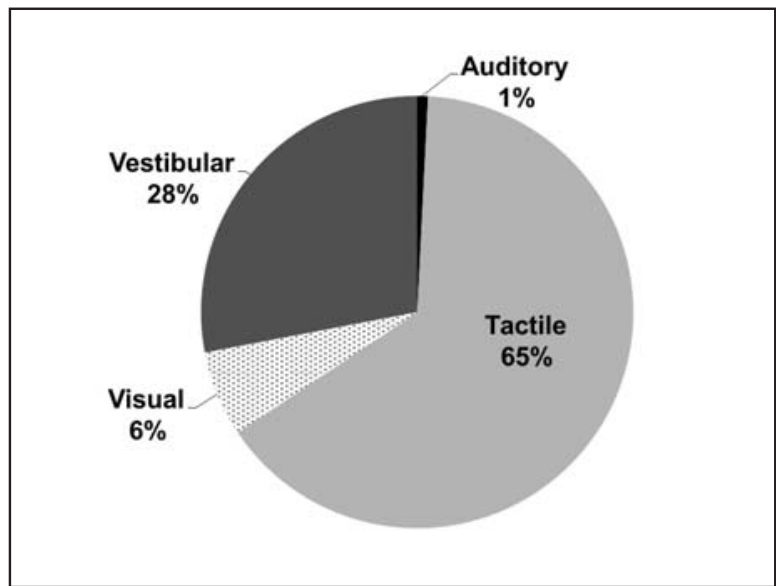

Fig 1: Frequency of behavioral responsesto different components of multisensory stimulation in preterm infants $(n=25)$.

\section{Discussion}

The primary aim of this descriptive study was to examine the occurrence and pattern of behavioral responses to multisensory stimulation in preterm infants over a period of 10 days. Limb activity and yawning were the frequently observed behavioral response to multisensory stimulation that decreased with successive stimulation sessions. These responses could indicate an improved alertness and habituation response to sensory stimulation in preterm infants. However, interpretation of these behaviors requires caution as they could indicate stress in preterm infants when coupled with other physiological and behavioral cues. Thus, sensory stimulation procedures in preterm infants requires a careful observation and interpretation of behavioral cues.

Limb activity (extension and flexion movement of arms and legs) was the most commonly observed behavior in this study. Occurrence of such motor activity 
is suggested to be associated with transition from sleep to wake state in preterm infants ${ }^{15}$. The second common behavior observed was yawning. Yawning has been shown to increase behavioral arousal in preterm infants between 30-35 weeks post-conceptual age and could play a role in stabilizing the drowsy state in preterm infants ${ }^{16}$.Such an improved alertness has shown to improve feeding performance and feeding efficacy in preterm infants. Moreover, yawning is also considered to indicate readiness for feeding in preterm infants ${ }^{17,18}$. The results from our study concur with previous studies where multisensory ATVV stimulation was found to be associated with improved alertness and feeding readiness behaviors such as yawning and improved feeding in stable preterm infants ${ }^{19}$. Whilst some authors consider motor activity such as single limb, multiple limb, gross body, head turn movements as a stress behavior and observed association with reduced oxygen saturation levels in preterm infants of 27 to 33 weeks gestational age ${ }^{20}$. However, preterm infants in our study did not show reduced oxygen saturation levels which could be due to better physiological stability of preterm infants at 33 weeks of gestational age when the sensory stimulations were performed.

Although yawning is reported as a behavior associated with feeding ${ }^{18}$, it could also be interpreted as a stress behavior, resulting from a challenged autonomic system ${ }^{2}$. While a particular behavioural cue may indicate a positive reponse in one preterm infant, it could indicate a negative response in another preterm infant depending on various factors. Hence, validation of behavioral cues in preterm infants is warranted as owing to the variations in behavioral responses due to individual differences in preterm infants ${ }^{20}$. Behavioral responses such as tongue protrusion, finger splay, startles, tremors, hiccups, crying were least observed (less than 10\%) in this study. Currently, interpretation of preterm behavioral responses to NICU environment or stimulation is difficult because lack of consistency in the behaviors reported in studies which could have been resulted from individual preterm differences such as gestational age, birth weight, associated comorbidities and frequent changes in physiological parameters. Moreover, variations in type, duration and intensity of environmental stressors may also influence the behavioral responses in preterm infants ${ }^{20}$. Further studies are required to better understand these behavioral responses and delineate them as positive or negative behaviors.

Majority of the behavioral responses in this study were observed during tactile stimulation suggesting preterm infants are more responsive to tactile stimulation as compared to auditory, visual or vestibular stimulation. This could be due to an increased maturity of tactile system as compared to other sensory systems. However, in this study interpretation of responses to stimulation of a particular sensory system is challenging due to interaction of one sensory system with another that could influence each other.

The multisensory stimulation program consisted of auditory, visual, tactile and vestibular stimulations. Recorded lullabies are used commonly in NICU owing to their beneficial effects on preterm infants ${ }^{21}$. The sound level of lullabies administered in our study was calibrated to $45-55$ decibels as it is recommended to support stable vital signs of infants in $\mathrm{NICU}^{22}$. Black and white pattern cards were used for visual stimulus for the reason that the preterm infants attend to these contrasts and could perceive $1 / 2$ inch stripes by 32 weeks of gestation ${ }^{23}$. Tactile stimulation was followed by vestibular stimulation as tactile stimulations have been reported to be too awakening and hence suggested to be followed by vestibular stimulation due to its modulating effects ${ }^{24}$.

One of the primary limitations of our study is small sample size due to it being a time bound study. The gestational age of the infants included in our study varied ranging from 28 to 36 weeks which could have potentially confounded the results. Nevertheless, this was addressed by assessing all the infants at 33-36 weeks, when the sensory systems are relatively more mature. Another confounding factor that could have influenced the behavioral responses of infants was prior exposure to handling or painful stimuli were not feasible to be controlled in the NICU setting. Furthermore, this study lacked a control group which could have enlightened our understanding of behavioral responses in comparison to typical responses of preterm infants. Lastly, in addition to stress behaviors, observations of self-regulatory behaviors could have improved the understanding of infants' coping behavior and therefore it warrants further research.

\section{Conclusion}

This study identified the behavioral responses of preterm infants to multisensory stimulation including auditory, tactile, visual, and vestibular stimulation. Majority of behavioral responses observed in our study were associated with tactile stimulation, with limb activity and yawning being the most frequently observed behaviors. These behaviors may be associated with improved alertness in preterm infants following multisensory stimulation. 


\section{References}

1. Holditch-Davis D, Blackburn S, VandenBerg $\mathrm{K}$. Newborn and infant neurobehavioral development. Comprehensive Neonatal Nursing: A Physiologic Perspective 3rd ed St Louis, MO 2003:272-3.

2. AlsH.Towardasynactivetheoryofdevelopment:Promise for the assessment and support of infant individuality. Infant Ment Health J 1982;3(4):229-43.http://dx.doi. org/10.1002/1097-0355(198224)3:4\%3C229::aidimhj2280030405\%3E3.0.co;2-h.

3. Grunau RE, Holsti L, Whitfield MF, Ling E. Are twitches, startles, and body movements pain indicators in extremely low birth weight infants? Clin J Pain 2000;16(1):37.http://dx.doi.org/10.1097/00002508200003000-00007.

4. Peters KL. Association between autonomic and motoric systems in the preterm infant. Clin Nurs Res 2001;10(1):82-90.

5. Peng NH, Bachman J, Jenkins $\mathrm{R}$, Chen $\mathrm{CH}$, Chang $\mathrm{YC}$, Chang YS, et al. Relationships between environmental stressors and stress biobehavioral responses of preterm infants in NICU. J Perinat Neonatal Nurs 2009;23(4):363.http://dx.doi.org/10.1097/ jpn.0b013e3181bdd3fd.

6. Burns K, Cunningham N, White-Traut R, Silvestri $\mathrm{J}$, Nelson MN. Infant stimulation: Modification of an intervention based on physiologic and behavioral cues. J Obstet Gynecol Neonatal Nurs 1994;23(7):581-9. http://dx.doi.org/10.1111/j.1552-6909.1994.tb01924.x.

7. White-Traut $R$, Wink $T$, Minehart $T$, Holditch-Davis D. Frequency of Premature Infant Engagement and Disengagement Behaviors During Two Maternally Administered Interventions. Newborn Infant Nurs Rev 2012;12(3):124-31.http://dx.doi.org/10.1053/j. nainr.2012.06.005.

8. Blackburn S. Environmental impact of the NICU on developmental outcomes. J Pediatr Nurs 1998;13(5):279-89.http://dx.doi.org/10.1016/s08825963(98)80013-4.

9. Smith GC, Gutovich J, Smyser C, Pineda R, Newnham C, Tjoeng $\mathrm{TH}$, et al. Neonatal intensive care unit stress is associated with brain development in preterm infants. AnnNeurol 2011.http://dx.doi.org/10.1002/ ana. 22545

10. Als $\mathrm{H}$. Developmental care in the newborn intensive care unit. Curr Opin Pediatr 1998;10(2):138.http:// dx.doi.org/10.1097/00008480-199804000-00004.

11. Sweeney JK, Heriza CB, Blanchard Y, Dusing SC. Neonatal physical therapy. Part II: Practice frameworks and evidence-based practice guidelines. Pediatr Phys Ther 2010;22(1):2-16.http://dx.doi.org/10.1097/ pep.0b013e3181cdba43.

12. Symington A, Pinelli J. Developmental care for promoting development and preventing morbidity in preterm infants. Cochrane Database Syst Rev 2006;2. http://dx.doi.org/10.1002/14651858.cd001814.pub2.

13. White-Traut RC, Nelson MN, Silvestri JM, Patel M, Vasan U, Kim Han B, et al. Developmental intervention for preterm infants diagnosed with periventricular leukomalacia. Res Nurs Health 1999;22(2):13143.http://dx.doi.org/10.1002/(sici)1098240x(199904)22:2\%3C131::aid-nur5\%3E3.0.co;2-e.

14. White-Traut RC, Nelson MN, Burns K, Cunningham N. Environmental influences on the developing premature infant: Theoretical issues and applications to practice. J Obstet GynecolNeonatal Nurs 1994;23(5):393-401. http://dx.doi.org/10.1111/j.1552-6909.1994.tb01896.x.

15. Thoman EB. Sleeping and waking states in infants: A functional perspective. Neurosci Biobehav Rev 1990;14(1):93-107.http://dx.doi.org/10.1016/s01497634(05)80165-4.

16. Giganti F, Hayes MJ, Akilesh MR, Salzarulo P. Yawning and behavioral states in premature infants. Dev Psychobiol 2002;41(3):289-96.http://dx.doi. org/10.1002/dev.10047.

17. Pickler RH, Best AM, Reyna BA, Wetzel PA, Gutcher GR. Prediction of feeding performance in preterm infants. Newborn Infant Nurs Rev 2005;5(3):116-23. http://dx.doi.org/10.1053/j.nainr.2005.04.001.

18. White-Traut RC, Berbaum ML, Lessen B, McFarlin $B$, Cardenas $L$. Feeding readiness in preterm infants: the relationship between preterm behavioral state and feeding readiness behaviors and efficiency during transition from gavage to oral feeding. Am J MaternChild Nurs 2005;30(1):52-9.

19. White-Traut RC, Nelson MN, Silvestri JM, Vasan U, Patel $\mathrm{M}, \mathrm{Cardenas} \mathrm{L}$. Feeding readiness behaviors and feeding efficiency in response to ATVV intervention. Newborn Infant Nurs Rev 2002;2(3):166-73.http:// dx.doi.org/10.1053/nbin.2002.35121.

20. Harrison LL, Roane C, Weaver M. The relationship between physiological and behavioral measures of stress in preterm infants. J Obstet Gynecol Neonatal Nurs 2004;33(2):236-45.http://dx.doi. org/10.1177/0884217504263293.

21. Standley JM. A meta-analysis of the efficacy of music therapy for premature infants. J Pediatr Nurs 2002;17(2):107-13.http://dx.doi.org/10.1053/ jpdn.2002.124128

22. Philbin MK, Robertson A, Hall III JW. Recommended permissible noise criteria for occupied, newly constructed or renovated hospital nurseries. $A d v$ Neonatal Care 2008;8(5):S11-S5.http://dx.doi. org/10.1097/01.anc.0000337267.47599.80.

23. Tecklin JS. Pediatric physical therapy, 3rd ed. Philadelphia: Lippincott Williams \& Wilkins; 2008.

24. White-Traut RC, Nelson MN, Silvestri JM, Cunningham $\mathrm{N}$, Patel M. Responses of preterm infants to unimodal and multimodal sensory intervention. Pediatr Nurs 1997;23(2):169-175. 